\title{
Perfil das Crianças Estomizadas em um Hospital Público de Teresina, Piauí
}

\author{
Profile of Stomized Children in a Public Hospital of Teresina, Piauí, Brazil
}

Perfil de Niños Estomizados en un Hospital Público de Teresina, Piauí, Brasil

Elaine Carininy Lopes da Costaํ, Daniele Silva do Vale², Maria Helena Barros Araújo Luz³

\begin{abstract}
RESUMO
As causas de estomias em crianças decorrem, principalmente, das más formações congênitas, obstruções intestinais e lesões em função de trauma. Esta pesquisa teve como objetivo descrever o perfil epidemiológico de crianças submetidas às estomias e a suas peculiaridades. Tratou-se de um estudo exploratório-descritivo, transversal de abordagem quantitativa, realizado em um hospital público de Teresina, que é referência estadual na assistência à saúde da criança e do adolescente. A coleta de dados foi realizada mediante análise de registro nos prontuários de pacientes atendidos no referido hospital correspondente a 2012 e 2013. Os dados foram organizados em planilha no Microsoft Excel, versão 2007, transposta para o Statistical Package for the Social Sciences para análise estatística descritiva. A pesquisa foi aprovada pelo o Comitê de Ética em Pesquisa da Universidade Federal do Piauí, sob o CAAE 012.1000922/13-64. Verificou-se que, das 59 crianças, a maioria é do sexo masculino e menor de dois anos, e a principal causa de confecção do estoma foi a malformação congênita, predominando as estomias gastrintestinais do tipo colostomia e gastrostomia, seguidas do sistema respiratório (traqueostomia) e do urinário (urostomias), corroborando com os resultados encontrados nas literaturas nacional e internacional. Destacam-se as lacunas de registros nos prontuários e a escassez de estudos relacionados às estomias em crianças. Dessa forma, esta pesquisa contribui para discutir a necessidade de planejamento e a efetiva assistência de Enfermagem à criança estomizada.
\end{abstract}

DESCRITORES: Estomas. Saúde da criança. Perfil.

\begin{abstract}
The causes of ostomies in children arise mainly from congenital malformations, intestinal obstructions, and trauma injuries. This research aimed at describing the epidemiological profile of children submitted to ostomy and its peculiarities. This is an exploratory-descriptive and cross-sectional study of a quantitative approach performed in a public hospital in Teresina, Brazil, a State reference in children and adolescents' health care. Data collection was performed by analyzing the medical records of patients receiving care in the hospital from 2012 to 2013. Data were organized in a Microsoft Excel 2007 spreadsheet, and they were transposed to the Statistical Package for the Social Sciences for descriptive statistical analysis. The study was approved by the Committee of Ethics in Research of the Federal University of Piauí with the CAAE 012.1000922/13-64. Among the 59 included children, most of them are male and aged less than two years. The leading cause of stoma confection was congenital malformation, and gastrointestinal ostomy of colostomy and gastrostomy type were more predominant, followed by the respiratory system (tracheostomy) and urinary system (urostomy), corroborating the results found in national and international literatures. There is an alarming focus upon the registry gaps in medical records and the lack of studies regarding ostomies in children. Thus, this research contributes to the planning need and to the effective Nursing care to the stomized child.
\end{abstract}

DESCRIPTORS: Stomata. Child health. Profile.

\footnotetext{
1Programa de Pós-graduação em Enfermagem da Universidade Federal do Piauí (UFPI) - Teresina (PI), Brasil. Endereço para correspondência:

Quadra 74 - Lote 04 - Casa A - Promorar - CEP: 64027-170 - Teresina (PI), Brasil - E-mail: ninyenfbio@yahoo.com.br

${ }^{2}$ Clínica e Maternidade Santa Fé - Teresina (PI), Brasil.

${ }^{3}$ Departamento de Enfermagem da UFPI - Teresina (PI), Brasil.

Artigo recebido em: 23/10/2015 - Aceito para publicação em: 09/03/2016
} 


\section{RESUMEN}

Las causas de estomas en niños se deben principalmente a las malformaciones congénitas, a las obstrucciones intestinales y las lesiones por trauma. Esta investigación tuvo como objetivo describir el perfil epidemiológico de los niños sometidos a la estomía y sus peculiaridades. Se trató de un estudio exploratorio-descriptivo, retrospectivo, con abordaje cuantitativo, realizado en un hospital público de Teresina, en Brasil, que es un estado de referencia en la asistencia a la salud de niños y adolescentes. La recolección de datos se realizó mediante el análisis de registro de las historias clínicas de los pacientes ingresados en el hospital citado correspondiente a los años 2012 y 2013 . Los datos fueron organizados en una hoja de cálculo en Microsoft Excel, versión 2007, transpuesta al Statistical Package for the Social Sciences para el análisis estadístico descriptivo. El estudio fue aprobado por el Comité de Ética en Investigación de la Universidad Federal de Piauí con el CAAE 012.1000922/13-64. Se encontró que entre los 59 niños, la mayoría son hombres con menos de dos años y la principal causa de confección de la estoma fue la malformación congénita, predominantemente gastrointestinal del tipo de colostomía y de gastrostomía, seguida del sistema respiratorio (traqueostomía) y del urinario (urostomía), corroborando los resultados encontrados en las literaturas nacional e internacional. Cabe destacar las ausencias de registros en los prontuarios y la falta de estudios relativos a la ostomía en los niños. Por lo tanto, esta investigación contribuye a la discusión de la necesidad de planificación y atención de Enfermería eficaz para el niño estomizado.

DESCRIPTORES: Estomas. Salud del niño. Perfil.

\section{INTRODUÇÃO}

A confecção de uma estomia em criança constitui uma medida terapêutica cirúrgica, de caráter provisório ou definitivo, para muitas doenças ou situações clínicas agudas ou crônicas. É uma situação que pode gerar alterações biopsicossociais, o que resulta na necessidade de cuidados à criança bem como aos pais, aos familiares e aos cuidadores ${ }^{1,2}$.

As causas de estomias em crianças decorrem, principalmente, de malformação congênita, obstruções intestinais e lesões decorrentes de trauma. No lactente, têm-se como causas das estomias a enterocolite necrosante, o ânus imperfurado e a doença de Hirschsprung; nas crianças maiores são as doenças inflamatórias intestinais e as ureterostomias para corrigir defeitos na bexiga e porção distal dos ureteres ${ }^{3-5}$.

Desse modo, a realização de uma estomia na criança, além de afetar a integridade corporal, a capacidade funcional, o convívio social e a qualidade de vida em geral, gera grande impacto em seu cotidiano e na dinâmica familiar ${ }^{5,6}$.

Nesse sentido, a criança deve ser avaliada como um ser em contínuo processo de crescimento e desenvolvimento, pois, ao apresentar alterações físicas, corporais ou comportamentais, pode ter sua personalidade violentada por meio da perda de segurança e dano ao próprio desenvolvimento, refletindo na vida adulta ${ }^{1}$.

Esta pesquisa foi realizada em um hospital público de referência na assistência à saúde da criança e do adolescente, em Teresina, Piauí, e tem como objetivo descrever os perfis sociodemográfico e epidemiológico, além das especificidades de estomias em crianças.
O estudo mostra-se relevante, pois as crianças estomizadas configuram-se como grupos de indivíduos extremamente vulneráveis que necessitam de apoio, acompanhamento e assistência especializada. Além disso, observou-se o quanto é escassa na literatura a quantidade disponível de informações sobre o tema e incipientes os estudos de caracterizações demográfica e clínica e de dados epidemiológicos acerca de causas das estomias.

A caracterização do perfil de pacientes/usuários dos serviços de saúde favorece a produção de dados epidemiológicos e clínicos que subsidiam o direcionamento e a adequação dos serviços, sendo importante para que os profissionais da equipe de saúde, envolvidos na assistência a uma determinada clientela, possam atender melhor às suas necessidades, promovendo maior segurança, satisfação e qualidade de vida.

\section{METODOLOGIA}

Trata-se de um estudo exploratório-descritivo, transversal, de abordagem quantitativa, que teve como critérios de inclusão os prontuários de crianças de 0 a 12 anos submetidas à confecção de uma estomia entre 2012 e 2013 no Hospital de referência à saúde da criança e adolescente no Estado do Piaui. Como critérios de exclusão, foram eliminados os prontuários com ausência de registros ou registros ilegíveis, bem como aqueles de crianças com idade acima de 12 anos.

A coleta de dados foi realizada mediante averiguação dos prontuários, utilizando um formulário pré-elaborado pelas autoras, no qual constavam as variáveis relacionadas 
ao sexo, à idade, à procedência da criança e às características dos estomas. Ao utilizar os critérios de inclusão e exclusão, obtiveram-se 62 prontuários, dos quais três foram excluídos em função de registros ilegíveis, ausência de informações importantes como a data de confecção da estomia ou dados pessoais, o que resultou numa amostra constituída por 59 prontuários. Os dados obtidos foram organizados em planilha do programa Microsoft Excel e, posteriormente, transpostos para o Statistical Package for the Social Sciences para análise utilizando estatística descritiva, com distribuição da frequência simples e percentual.

A pesquisa foi aprovada pelo Comitê de Ética em Pesquisa da Universidade Federal do Piauí, protocolo CAAE.012.1000922/13-64, e desenvolvida conforme a Resolução 466/12, do Conselho Nacional de Saúde 7 .

\section{RESULTADOS}

Foram selecionados para análise 59 prontuários de crianças estomizadas no período de 2013 e 2014, em que a maioria (34/59; 57,7\%) realizou o procedimento cirúrgico em 2013 e os demais $(25 / 59 ; 42,3 \%)$, em 2012. Do total de participantes, $33(55,9 \%)$ eram do sexo masculino e 49 (83\%) estavam na faixa etária entre 0 e 2 anos; 6 (10,2\%), entre 3 e 5 anos e $4(6,8 \%)$, entre 6 e 12 anos. Quase a totalidade era da cor parda (58/59; 98,3\%). A maioria dos participantes era procedente de interior do Piauí (41/59; 69,5\%); 10 (16,9\%) eram de Teresina e $8(13,6 \%)$ de outros estados (Tabela 1$)$.

Quanto à etiologia das estomias, foram encontradas 63 causas básicas relacionadas, das quais $38(64,40 \%)$ foram por malformação congênita; 20 (33,90\%) eram patologias diversas associadas, como insuficiência respiratória, obstruções uretrais, neoplasias, dentre outros; e 5 (8,47\%) foram causadas por trauma.

Foram identificados 66 diagnósticos médicos relacionados à indicação da estomia nas 59 crianças, dentre os quais: anomalia anorretal (26/59; 39\%); megacólon congênito e insuficiência respiratória aguda corresponderam a sete (11\%) cada uma; doença do refluxo gastresofágico (6/59; $9 \%)$; perfuração intestinal (3/59; 4\%); atresia do esôfago $(3 / 59 ; 4 \%)$ e outros diagnósticos (14/59;21\%). Estes, por sua vez, foram: bexiga neurogênica, estenose uretral, estenose cáustica do esôfago, laringotraqueomalácia, obstrução uretral, válvula da uretra posterior, peritonite aguda, polipose adenomatosa familiar, estenose subglótica e papilomatose laríngea (Gráfico 1).
Tabela 1. Caracterização das crianças submetidas a estomias conforme ano de internação, sexo, faixa etária, cor e procedência. Teresina (PI), 2014.

\begin{tabular}{|c|c|c|}
\hline Características & $\mathrm{n}$ & $\%$ \\
\hline \multicolumn{3}{|l|}{ Ano de internação } \\
\hline 2013 & 34 & 57,7 \\
\hline 2012 & 25 & 42,3 \\
\hline \multicolumn{3}{|l|}{ Sexo } \\
\hline Masculino & 33 & 55,9 \\
\hline Feminino & 26 & 44,1 \\
\hline \multicolumn{3}{|l|}{ Faixa etária (anos) } \\
\hline 0 a 2 & 49 & 83,0 \\
\hline 3 a 5 & 6 & 10,2 \\
\hline 6 a 12 & 4 & 6,8 \\
\hline \multicolumn{3}{|l|}{ Cor } \\
\hline Branco & 1 & 1,7 \\
\hline Pardo & 58 & 98,3 \\
\hline \multicolumn{3}{|l|}{ Procedência } \\
\hline Interior do Piauí & 41 & 69,5 \\
\hline Teresina & 10 & 16,9 \\
\hline Outros estados & 8 & 13,6 \\
\hline Total & 59 & 100,00 \\
\hline
\end{tabular}

Fonte: Prontuários do Serviço de Arquivo Médico e Estatística do Hospital.

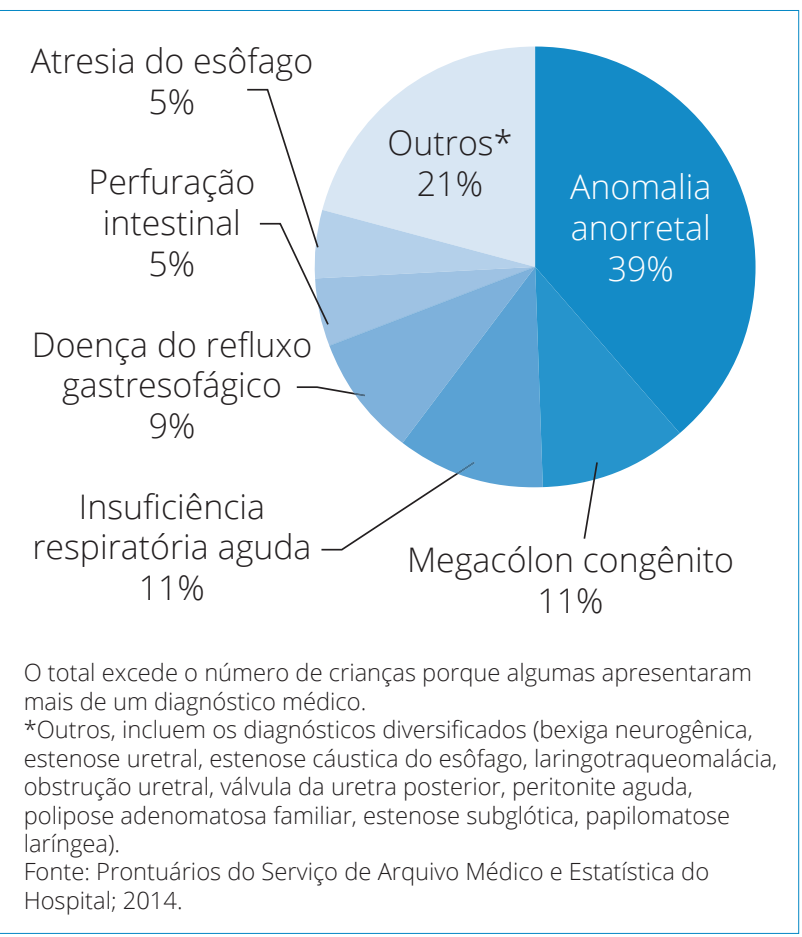

Gráfico 1. Distribuição dos diagnósticos médicos das crianças estomizadas $(n=59)$, Teresina $(\mathrm{PI})$. 
Verificaram-se diversos sistemas orgânicos envolvidos nos tipos de estomias confeccionados, nas 59 crianças, totalizando 65 estomias. Destas, a maioria (50/59; 84,74\%) foi realizada no sistema digestório, distribuída da seguinte forma: $33(55,93 \%)$ colostomias; $11(18,64 \%)$ gastrostomias, 5 (8,47\%) ileostomias e uma (1,69\%) esofagostomia. Em relação ao sistema respiratório, foram $8(13,55 \%)$ traqueostomias e ao sistema urinário foram 7 (11,86\%) cistostomias (Tabela 2).

Tabela 2. Distribuição dos tipos de estomas conforme sistema orgânico, tempo de internação e complicações apresentadas pelas crianças participantes $(\mathrm{n}=59)$. Teresina (PI), 2014.

\begin{tabular}{|c|c|c|}
\hline Características & $\mathrm{n}$ & $\%$ \\
\hline \multicolumn{3}{|l|}{ Sistema orgânico acometido } \\
\hline \multicolumn{3}{|l|}{ Sistema digestório $(n=50)$} \\
\hline Colostomia & 33 & 55,93 \\
\hline Gastrostomia & 11 & 18,64 \\
\hline Ileostomia & 5 & 8,47 \\
\hline Esofagostomia & 1 & 1,69 \\
\hline \multicolumn{3}{|l|}{ Sistema respiratório $(\mathrm{n}=8)$} \\
\hline Traqueostomia & 8 & 13,55 \\
\hline \multicolumn{3}{|l|}{ Sistema urinário (n=7) } \\
\hline Cistostomia & 7 & 11,86 \\
\hline Total & $65^{*}$ & 100 \\
\hline \multicolumn{3}{|c|}{ Tempo de internação (dias) } \\
\hline 1 a 15 & 38 & 64,4 \\
\hline 15 a 30 & 13 & 22,0 \\
\hline$>30$ & 8 & 13,6 \\
\hline \multicolumn{3}{|l|}{ Complicações } \\
\hline Não & 50 & 84,7 \\
\hline $\operatorname{Sim}$ & 9 & 15,2 \\
\hline Total & 59 & 100 \\
\hline \multicolumn{3}{|l|}{ Tipos de complicações } \\
\hline Estenose & 4 & 44,45 \\
\hline Prolapso & 4 & 44,45 \\
\hline Dermatite periestoma & 1 & 11,10 \\
\hline Total & 9 & 100 \\
\hline
\end{tabular}

*O total excede o número de crianças porque algumas apresentaram estomas em mais de um sistema.

Fonte: Prontuários do Serviço de Arquivo Médico e Estatística do Hospital.
No que se refere ao tempo de internação, $38(64,4 \%)$ permaneceram hospitalizadas por um período de 1 a 15 dias; $13(22 \%)$, de 15 a 30 dias; e $8(13,6 \%)$ por mais de 30 dias. Quanto à ocorrência de complicações relacionadas à confecção da estomia, notou-se que $9(15,25 \%)$ apresentaram complicações, sendo $4(44,45 \%)$ estenoses, $4(44,45 \%)$ prolapsos e 1 (11,11\%) dermatite periestoma (Tabela 2).

\section{DISCUSSÃO}

Das 59 crianças participantes do estudo, a maioria confeccionou o estoma em 2013, pertencia ao sexo masculino e estava na faixa etária de zero a dois anos, a que se refere a recém-nascidos e lactentes. A cor predominante foi a parda. Grande parte das crianças era procedente de cidades do interior do Piauí. Os resultados obtidos referentes ao sexo e à faixa etária são semelhantes aos encontrados no Distrito Federal, em que houve um maior número de crianças do sexo masculino (62\%) e da faixa etária de 0 a 5 anos (84\%) Outra pesquisa no Brasil com crianças estomizadas mostrou predomínio do sexo masculino com $66,7 \% \%^{5}$.

É importante ressaltar que havia 59 crianças com 66 diagnósticos, haja vista que algumas apresentavam mais de um diagnóstico médico. A confecção de estomias em Pediatria, geralmente, é atribuída à malformações congênitas, cujo procedimento é normalmente realizado no período neonatal. O diagnóstico mais frequente foi anomalia anorretal com 26 casos, correspondendo a 39\% das crianças. Corroborando com estes resultados, um estudo realizado em Recife com crianças estomizadas obteve a anomalia anorretal como a principal causa para confecção da estomia $(53,2 \%)$, seguida do megacólon congênito $(13,3 \%)^{9}$. Esses resultados se diferem dos achados encontrados no estudo no Distrito Federal, em que a principal etiologia para a confecção de estomias foi o megacólon congênito (48\%), seguido das anomalias anorretais $(11 \%)^{8}$.

As anomalias anorretais (AAR) são desordens que ocorrem em cerca de 1 em 1.500 a 1 em 5.000 nascidos vivos em todo o mundo. São consideradas anomalias congênitas caracterizadas pela ausência de ânus, cujos transtornos, geralmente, requerem intervenções cirúrgicas no período neonatal, acompanhamento pós-operatório e tratamento para obter e manter a continência fecal ${ }^{10,11}$.

Ao considerar os diagnósticos para a confecção do estoma, observam-se, neste estudo, resultados semelhantes 
aos encontrados na literatura específica, em que a principal causa para realizar estomas em crianças e adolescentes é a malformação congênita, seguida dos problemas patológicos como enterocolite necrotizante, câncer e eventos traumáti$\cos ^{5,8,9}$. Como as causas responsáveis pela confecção de estomias em crianças geralmente estão associadas à malformação congênita, observa-se uma maior frequência no caráter temporário das estomias, pois possuem o objetivo de proteger uma sutura ou anastomose distal, facilitando assim a posterior reconstrução do trânsito intestinal ${ }^{8}$. No presente estudo, não foi possível determinar o caráter de permanência das estomias, uma vez que não foram encontradas informações suficientes relacionadas a essa variável nos prontuários.

O sistema orgânico mais frequentemente acometido foi o digestório, seguido do respiratório e do urinário, resultado semelhante a outras pesquisas em que o sistema digestório foi o mais frequente ${ }^{5,8,12}$.

Com relação ao tipo de estomia, a colostomia apresentou-se como a mais frequente, ocorrendo em 33 (55,93\%) participantes do estudo. $\mathrm{O}$ segundo tipo com maior ocorrência foi a gastrostomia com $11(18,64 \%)$ casos, seguida da traqueostomia, com 8 (13,55\%). Um estudo que descreveu o perfil de crianças e adolescentes estomizados no Distrito Federal observou que $67,6 \%$ dos participantes eram colostomizados, $23,2 \%$ ileostomizados e 7,12\% tinham outros tipos de estomia ${ }^{8}$. O predomínio da colostomia em relação aos outros tipos de estomias assemelha-se aos resultados encontrados em outros estudos brasileiros com crianças estomizadas ${ }^{9,13}$.

Quanto ao tempo de internação, observou-se que a maioria dos participantes permaneceu internada no intervalo entre 1 e 15 dias, porém constatou-se a presença de crianças internadas durante mais de 30 dias. Tal ocorrência se deu em consequência das complicações relacionadas ao estoma, já que nove $(15,25 \%)$ crianças apresentaram-nas, sendo que as mais frequentes foram: a estenose e o prolapso e a menos frequente foi a dermatite periestoma. Corroborando com estes resultados, um estudo realizado na Turquia com 157 crianças estomizadas acometidas por anomalia anorretal obteve uma taxa de complicação semelhante $(15,2 \%)$, e as complicações de maior ocorrência foram o prolapso $(4,4 \%)$ e a estenose $(3,8 \%)^{14}$.

É importante ressaltar que a presença de uma estomia de eliminação intestinal, em uma criança, representa o rompimento de sua integridade física e funcional frente a sua fragilidade e necessidades específicas do crescimento e desenvolvimento.
Assim, exige-se do enfermeiro um cuidado efetivo na promoção da saúde e na prevenção de agravos a fim de que se possa favorecer a obtenção de segurança e qualidade de vida.

O enfermeiro assume um importante papel no preparo dos familiares e dos cuidadores para realizarem o cuidado efetivo da criança estomizada, por meio do ensino e do desenvolvimento de novas habilidades, de orientações quanto à higiene dos estomas, da identificação de aspectos relacionados a normalidades e manifestações precoces de alterações, tornando-os capacitados a desenvolver ações preventivas e de integração social e reabilitação ${ }^{15}$.

As ações de prevenção consistem em orientar os estomizados, bem como os seus cuidadores e familiares, a respeito dos cuidados com o estoma e a pele ao redor do estoma, de demonstração da retirada e colocação dos equipamentos coletores e da higienização das bolsas a fim de evitar complicações ${ }^{15}$.

\section{CONCLUSÃO}

O estudo permitiu caracterizar o perfil de crianças entre 0 e 12 anos submetidas à estomia entre 2012 e 2013 em um hospital público de referência em Pediatria, no município de Teresina. A amostra foi constituída por 59 prontuários de crianças estomizadas, a maioria do sexo masculino, na faixa etária entre 0 e 2 anos, cuja principal causa para confecção do estoma foi a malformação congênita relacionada ao sistema gastrintestinal, sendo a colostomia o tipo de estoma mais frequente.

A assistência ao cliente estomizado, em especial à criança, requer abordagem interdisciplinar e conhecimentos por parte dos profissionais de saúde de modo a abranger a mãe, a família e os cuidadores para promover uma atenção integral e humanizada, levando em consideração as necessidades do indivíduo e das pessoas envolvidas, sejam elas físicas, emocionais ou psicossociais. Vale ressaltar que estudos da análise de perfil são importantes, pois fornecem subsídios com a finalidade de guiar o planejamento da assistência, mediante a incorporação dos fundamentos necessários à equipe multiprofissional de características da criança estomizada.

Foi possível perceber também uma pequena quantidade de estudos relacionados às estomias e à infância e as diversidades de diagnósticos médicos e de tipos de estomas envolvidos. Assim, vale ainda destacar as dificuldades e limitações do estudo em função da falta de registros nos 
prontuários e da escassez de referências na literatura nacional e internacional sobre esta temática. Espera-se que este estudo possa contribuir para o planejamento da assistência de Enfermagem à criança estomizada, bem como servir de subsídios para novos estudos sobre este tema.

\section{AGRADECIMENTOS}

Agradecemos ao Conselho de Desenvolvimento Científico e Tecnológico (CNPq) pelo suporte financeiro concedido no período de 2013-2014 para realização da pesquisa.

\section{REFERÊNCIAS}

1. Barreire SG, Oliveira OA, Kazama W, Kimura M, Santos VL. Qualidade de vida de crianças ostomizadas na ótica das crianças e das mães. J Pediatr. 2003;79(1):55-62.

2. Santos VL, Cesaretti IU. Assistência em estomaterapia: cuidando do ostomizado. SãoPaulo: Atheneu; 2005.

3. Silva DM. Assistência de Enfermagem à criança com estomia no trato digestório. [Dissertação]. Universidade de Brasília, Faculdade de Ciências da Saúde, Programa de Pós-Graduação em Enfermagem. 2013. [citado 14 jan. 1016]. Disponível em: http://repositorio.unb.br/bitstream/10482/14789/1/2013_ DanielaMoniciDaSilva.pdf

4. Melo MC, Kamada I. Anomalia anorretal e cuidados maternos. Rev Bras Enferm. 2011;64(1):176-9.

5. Valdes AG, Kamada I, Cristo RC, Costa SB, Faustino AM. Experiências decriançascomestomias: estudo qualitativo. Rev Estima. 2010;8(3):19-26. [citado 14 jan. 2016]. Disponível em: http://www.revistaestima.com.br/index.php?option=com_ content\&view=article\&id=36:artigo-original-2\&catid=16: edicao-vol-83\&ltemid=87

6. Delavechia RP, Terra MG, Noal HC, Padoin SM, Lacchini AJ, Silva ME. A percepção de si como ser-estomizado: um estudo fenomenológico Rev Enferm UERJ. 2010;18(2):223-8. [citado 14jan. 2016]. Disponível em: http://wnw.facenf.uerj.br/v18n2/v18n2a10.pdf

7. Brasil. Ministério da Saúde. Diretrizes e normas regulamentadoras de pesquisas envolvendo seres humanos: resolução n 466, de 12 de dezembro de 2012. República Federativa do Brasil. Brasília (DF); 2012.

8. Monteiro SN, Kamada I, Silva AL, Souza TC. Perfil de crianças e adolescentes estomizados atendidos de um Hospital Público do Distrito Federal. Rev Estima. 2014;12(3):23-32. [citado 28 nov. 2015]. Disponível em: http://www.revistaestima.com.br/ index.php?option=com_content \&view=article\&id=484\%3A artigo-original- 2\&catid=49\%3A2014-12-01-16-18-37\&ltemid= 109\&lang=pt

9. Egito ET, Medeiros AQ, Moraes MM, Barbosa JM. Estado nutricional de pacientes pediátricos ostomizados. Rev Paul Pediatr. 2013;31(1):58-64.
10. Wijers $\mathrm{CH}$, Blaauw I, Marcelis $\mathrm{CL}$, Wijnen RM, Brunner $\mathrm{H}$, Midrio $\mathrm{P}$, et al. Research perspectives in the etiology of congenital anorectal malformations using data of the International Consortium on Anorectal Malformations: evidence for risk factors across different populations. Pediatr Surg Int. 2010;26(1):1093-9. [citado 18 out. 2014]. Disponível em: http://www.ncbi.nlm.nih.gov/pmc/articles/ PMC2962787/pdf/383_2010_Article_2688.pdf

11. Goossens WJ, Blaauw I, Wijnen MH, Gier RP, Kortmann B, Feitz WF. Urological anomalies in anorectal malformations in The Netherlands: effects of screening all patients on long-term outcome. Pediatr Surg Int. 2011;27(1):1091-7. [citado 18 out. 2014]. Disponível em: http://www.ncbi.nlm.nih.gov/pmc/articles/ PMC3175030/pdf/383_2011_Article_2959.pdf

12. Melo MC. Experiência materna com o filho estomizado. [Dissertação]. Universidade de Brasília, Faculdade de Ciências da Saúde, Programa de Pós-Graduação em Enfermagem. Brasília; 2010. [citado 14 jan. 2016]. Disponível em: http:// repositorio.unb.br/bitstream/10482/7044/1/2010_ ManuelaCostaMelo.pdf

13. Menezes HF, Goés FG, Maia SM, Souza AL. A subjetividade no cuidado familiar à criança ostomizada a partir da construção da sua autonomia. Rev Pesq Cuid Fundam Online. 2013;5(2):3731-9. [citado 14 jan. 2016]. Disponível em: http:// www.ssoar.info/ssoar/bitstream/handle/document/34425/ ssoar-revpesquisa-2013-2-menezes_et_al-Subjectivity_in_ family_care_for.pdf?sequence $=1$

14. Dermirogullari B, Yilmaz Y, Yildiz GE, Ozen IO, Karabulut R, Turkyilmaz Z, et al. Ostomy complicatıons in patients with anorectal malformations. Pediatr Surg Int. 2011;27:1075-8. [citado 18 out. 2014]. Disponível em: http://link.springer. com/article/10.1007/s00383-011-2955-8.

15. Mauricio VC, Lisboa MT, Souza NV. O enfermeiro e sua participação no processo de reabilitação da pessoa com estoma. Esc Anna Nery. 2013;17(3):416-22. 